\title{
Habilidades procedimentales en Cálculo Diferencial desarrolladas por la interacción entre pares académicos
}

\author{
Ana Milena Santamaría Bueno \\ Sandra Evely Parada Rico
}

Universidad Industrial de Santander

e-mail: a.m.santamaria10@gmail.com

\begin{abstract}
Resumen
El objetivo de la investigación que aquí se reporta es identificar y describir habilidades que desarrollan estudiantes beneficiarios de un programa de acompañamiento y seguimiento de Cálculo Diferencial para elaborar, comparar y ejercitar procedimientos. Para identificar dichas habilidades nos centraremos en la Taxonomía de los procedimientos de Barajas y en la interpretación del programa como como una Zona de Desarrollo Próximo según Vygotsky. Presentamos el análisis de un caso de estudio que fue beneficiario de los proyectos que el programa ofrece a los estudiantes de primer semestre de la Universidad Industrial de Santander. Se identifica un avance en los procedimientos analíticos del caso de estudio para la resolución de problemas de fenómenos variacionales.
\end{abstract}

\section{Introducción}

Desde la década de los noventa se ha evidenciado el interés por parte de algunos investigadores, como [16] y [6] en profundizar en la enseñanza y el aprendizaje de temas relacionados con el Cálculo Diferencial e Integral. Al respecto, [5] y [1] han indagado sobre los errores, dificultades y conocimientos en matemáticas de los estudiantes de los últimos años de secundaria y de los primeros cursos universitarios, tomando así fuerza la línea del Pensamiento Matemático Avanzado.

Con respecto a los conocimientos en matemáticas los documentos orientadores del currículo plantean que los estudiantes al terminar la formación preuniversitaria deben ser capaces de comprender relaciones y funciones, seleccionando y utilizando varias formar de representarlas, así como analizar el cambio en contextos diversos ([13], [10], [11]). Sin embargo, algunos autores $([1],[9],[7],[3])$ plantean que los estudiantes que ingresan a la educación superior no traen los conocimientos y habilidades necesarios de álgebra y pre-cálculo para afrontar, en particular, un curso de cálculo diferencial. Aludiendo a que a pesar de hacer énfasis en el desarrollo de procesos la prioridad está en el aprendizaje de conceptos ([7]) perdiéndose el equilibrio entre procesos y contenidos matemáticos.

Esta problemática se ve reflejada en las cifras de reprobación de los cursos de cálculo diferencial; en particular, la Universidad Industrial de Santander (UIS) tiene una problemática de deserción y de reprobación de dicha asignatura, con cifras entre el 49 y $64 \%$ de reprobación (Vicerrectoría Académica, 2011). Así, en 2012 la UIS implementó el programa de Atención, Seguimiento y Acompañamiento a Estudiantes (ASAE), contexto de la investigación, que 
junto con otras alternativas hace parte del Sistema de Excelencia Académica (SEA). El programa ofrece apoyo principalmente a estudiantes de primer nivel en el curso de Cálculo I y tiene las siguientes características: 1. Al estudiante beneficiario se le asigna un tutor durante todo el semestre para trabajar con él dos horas a la semana; 2. El programa está orientado en la resolución de problemas y en el desarrollo de procesos matemáticos; 3. Los tutores deben cumplir los requisitos de ser estudiantes de Licenciatura en Matemáticas y cursar la asignatura de didáctica del cálculo.

El programa reporta semestralmente las cifras de los resultados académicos que dan cuenta de un impacto positivo a nivel cuantitativo. No obstante, más que la parte cuantitativa consideramos necesario un estudio cualitativo desde la Educación Matemática para revisar el impacto que el programa tiene en los estudiantes beneficiarios. Por ello el estudio que aquí reportamos tiene la siguiente pregunta de investigación: ¿qué habilidades desarrollan estudiantes beneficiarios de un programa de acompañamiento y seguimiento de Cálculo Diferencial para elaborar, comparar y ejercitar procedimientos? En este sentido ubicamos la investigación en la línea del Pensamiento Matemático Avanzado.

Para dar respuesta a la pregunta coordinamos la taxonomía de los procedimientos de [3] con elementos teóricos de la teoría del aprendizaje de Vygotsky. Con el primer referente buscamos clasificar las habilidades procedimentales de los estudiantes, y con el segundo analizaremos las interacciones entre el tutor y el estudiante en el desarrollo de dichas habilidades.

En lo que sigue, describiremos los referentes teóricos utilizados en nuestra investigación, el diseño metodológico adoptado, los resultados obtenidos de un caso de estudio y conclusiones parciales que se derivan del análisis.

\section{Referentes teóricos}

2.1 Pensamiento Variacional y el proceso de elaboración comparación y ejercitación de procedimientos

Dentro de la estructura curricular colombiana, el pensamiento matemático se subdivide en cinco tipos de pensamiento denominados así: i. pensamiento numérico; ii. pensamiento espacial; iii. pensamiento métrico; iv. pensamiento aleatorio; y, vi. pensamiento variacional. Este último tipo de pensamiento, necesario en cálculo diferencial, será entendido a partir de lo que expone el Ministerio de Educación Nacional (MEN):

... este tipo de pensamiento tiene que ver con el reconocimiento, la percepción, la identificación y la caracterización de la variación y el cambio en diferentes contextos, así como con su descripción, modelación y representación en distintos sistemas o registros simbólicos, ya sean verbales, icónicos, gráficos o algebraicos. (p. 66)

De lo anteriormente citado y teniendo presente que los procesos matemáticos mencionados por el [10] son cinco: i) razonamiento; ii) comunicación; iii) modelación; iv) elaboración, comparación y ejercitación de procedimientos; y v) resolución y planteamiento de problemas, se considera dentro de nuestra investigación al proceso de resolución y planteamiento de 
problemas como el gran proceso dentro del cual se encuentran lo demás, por su estrecha relación con cada uno de ellos ([15]). Para la investigación se revisará lo que se entiende por el proceso de elaboración, comparación y ejercitación de procedimientos que dentro del trabajo será renombrado como proceso procedimental.

Ahora, retomamos lo que [15] han conceptualizado y contextualizado para el campo de la educación matemática sobre habilidad:

consideraremos aquí que una habilidad puede ser un conjunto de acciones secuenciales coherentes y coordinadas realizadas por un individuo, en la consecución de un objetivo. Estas acciones están mediadas por los conocimientos previos y pueden desarrollarse mediante la práctica. (p. 6)

Con respecto al proceso en el cual nos enfocaremos, identificamos que [3] logra la caracterización del proceso de Elaboración, comparación y ejercitación de procedimientos, específicamente para el Pensamiento Variacional, y a su vez la autora plantea una clasificación de los procedimientos en cuatro tipos: aritmético, geométrico, métrico y analítico, y propone unas habilidades a priori (1) para el este pensamiento por medio de descriptores: "expresiones verbales escritas, relacionadas con la habilidad y que tiene como fin contribuir a describir las actuaciones de los estudiantes en las diferentes tareas variacionales" (p. 45).

Tabla 1: Descriptores de las habilidades a priori para el pensamiento variacional.

\begin{tabular}{|l|l|}
\hline Procedimiento & Descriptores \\
\hline \multirow{5}{*}{ Aritmético } & $\checkmark$ Realizar, con los números reales, operaciones básicas y superiores. $\left(F_{2}\right)$ \\
& $\checkmark$ Usa diferentes notaciones de los números reales y establece relaciones para \\
& decidir sobre su uso en una situación dada. $\left(F_{3}\right)$ \\
& $\checkmark$ Establece relaciones que involucran números naturales y utiliza propiedades de \\
& los números para justificarlas. $\left(F_{1}\right)$ \\
\hline \multirow{3}{*}{ Métrico } & $\checkmark$ Emplea correctamente los aparatos de medida más comunes de las magnitudes \\
& longitud, tiempo, amplitud, capacidad, peso y superficie. ( $\left.F_{1} 0\right)$ \\
& $\checkmark$ Domina el sistema métrico decimal. $\left(F_{9}\right)$ \\
\hline \multirow{5}{*}{ Geométrico } & $\checkmark$ Realiza representaciones en el plano. $\left(F_{4}\right)$ \\
& $\checkmark$ Emplea un procedimiento de tipo gráfico que supone expresar una imagen \\
& visual de un concepto o relación variacional. $\left(F_{5}\right)$ \\
& $\checkmark$ Modela fenómenos periódicos del mundo real usando relaciones y funciones \\
& trigonométricas. \\
\hline \multirow{5}{*}{ Analítico } & $\checkmark$ Determina las variables de una situación. $\left(F_{12}\right)$ \\
& $\checkmark$ Establece correctamente la interdependencia de las magnitudes variables. $\left(F_{13}\right)$ \\
& $\checkmark$ Representa situaciones de cambio a través de las funciones, las gráficas y las \\
& tablas. $\left(F_{8}\right)$ \\
& $\checkmark$ Traduce de una a otra de las distintas representaciones de una función. \\
& $\checkmark$ Relaciona las expresiones algebraicas y gráfica empleando sus propiedades. $\left(F_{6}\right)$ \\
& $\checkmark$ Determina procesos infinitos que subyacen en las notaciones decimales. $\left(F_{7}\right)$ \\
\hline
\end{tabular}

La abreviatura alfa-numérica al final de cada descriptor, en la Tabla 1, hace referencia al código con el que se identifica dentro del instrumento de seguimiento que diseñamos y mencionamos en la metodología. 


\subsection{Elementos teóricos del aprendizaje según Vygotsky}

Vygotsky estudió los procesos psicológicos superiores del ser humano, como por ejemplo el lenguaje que inicialmente no nos pertenecen, sino que pertenecen al grupo humano en el que nacemos, así una de sus premisas es que estos procesos se originan en la vida social, es decir, en la interacción del sujeto con otros en la realización de actividades. El lenguaje como proceso es considerado fundamental ya que se convierte en una de las principales herramientas de aprendizaje, lo que nos lleva a confirmar que el diálogo continuo favorece la construcción de conocimiento.

En ese sentido al hablar de diálogo e interacción social para posibilitar el aprendizaje nos trae a colación el tipo de aprendizaje colaborativo, dado que son características esenciales presentes en este tipo de aprendizaje como lo plantean [8]. Para estos autores este aprendizaje se propone como un proceso social de construcción de conocimiento, en donde al establecer metas comunes se propicia reciprocidad entre el conjunto de individuos.

Ahora bien, uno de los elementos que mayor repercusión tiene en el análisis de las prácticas educativas es la denominada Zona de Desarrollo Próximo (ZDP); seguido de otros elementos como el andamiaje, y procesos de interiorización.

La definición de ZDP más usada y trabajada por los investigadores que proviene de la formulación original en la obra de Vygotsky es la siguiente:

la distancia entre el nivel real de desarrollo, determinado por la capacidad de resolver independientemente un problema, y el nivel de desarrollo potencial, determinado a través de la resolución de un problema bajo la guía de un adulto o en colaboración con otro compañero más capaz. ([2] p. 137)

En donde consideramos que en el nivel de desarrollo real se encuentran los conocimientos necesarios para solucionar un problema, en nuestro caso de matemáticas, y que además permiten con la ayuda de un experto resolver nuevos problemas con un grado de complejidad mayor. [14] menciona que la importancia de la ZDP consiste en que destaca la diferencia entre el desarrollo real y el desarrollo potencial, enfatiza en el valor de la actividad de colaboración y de la comunicación en el proceso de enseñanza así como el papel del profesor como orientador y favorecedor de ese proceso (p. 15). De esta forma se puede interpretar que la ZDP no se limita únicamente a lo que el estudiante puede o no hacer independientemente, sino a una estructura mayor y más allá del sujeto, como lo plantea [2] al decir que "la ZDP obliga a pensar más que en una capacidad o característica de un sujeto, en las características de un sistema de interacción socialmente definido" (p. 138). En esos términos la ZDP se puede entender como un "sistema social" y llevándolo al contexto local de la investigación entonces el programa de acompañamiento siendo un sistema social innato se puede interpretar desde este elemento teórico.

Se plantea que para que el estudiante adquiera autonomía en el desarrollo de los problemas una de las cosas a tener en cuenta es que el apoyo que brinda esa persona con mayor dominio debe tener ciertas características; sin embargo Vygotsky no lo planteó explícitamente, pero otros autores como Woods, Bruner y Ross en 1976 hicieron la formulación del andamiaje, que rápidamente se asoció con la ZDP según [2].

El termino andamiaje se entiende como una situación de interacción entre un sujeto experto, o más 
experimentado en un dominio, y otro novato, o menos experto, en la que el formato de la interacción tiene por objetivo que el sujeto menos experto se apropie gradualmente del saber experto. (p. 148)

De acuerdo a esto el andamiaje está inmerso en la ZDP y algunas recomendaciones para que la situación de interacción sea óptima es que el novato participe desde el comienzo en la actividad que reconoce como compleja, sin importar que su participación inicial sea sobre aspectos parciales de la actividad global. Por lo tanto, la idea de andamiaje hace referencia a que la actividad se resuelve "colaborativamente". Por otra parte, haciendo la analogía con los andamios en construcción, este elemento debe ser ajustable, temporal, audible y visible $([2])$.

Por último el proceso de interiorización es entendido como el proceso de pasar de lo interpsiquico a lo intrapsiquico, es decir de lo creado socialmente, el conocimiento colectivo al pensamiento y conocimiento autónomo del sujeto; que en últimas conlleva a la apropiación de lo aprendido socialmente para actuar por sí mismo. Este proceso se puede identificar en el trabajo explícito dentro de la sesión de tutoría y la actividad complementaria que el estudiante debe realizar después de ella y que es propiciada por el tutor.

Teniendo en cuenta las ideas mencionadas, y con la caracterización del programa de acompañamiento y seguimiento, como espacio de interacción continua entre el tutor y el estudiante, decidimos analizar e interpretar las situaciones de aprendizaje que se evidencian en las sesiones de tutoría desde los elementos teóricos ya expuestos.

\section{$3 \quad$ Metodología}

La investigación adoptó un proceso metodológico organizado hasta el momento en seis fases que se describen a continuación:

Fase 1. Estudio preliminar. Esta fase corresponde a la recolección de datos y análisis cuantitativo del rendimiento académico, en los distintos cursos de cálculo (Cálculo I, II, III y Ecuaciones Diferenciales), de los estudiantes que han participado en el programa desde 2013I. Este estudio ya fue realizado gracias a la información proporcionada por la Vicerrectoría Académica de la Universidad. Y los resultados obtenidos fueron presentados por Santamaría y Parada (2015). En particular, se compararon las cifras de aprobación de los estudiantes beneficiarios de ASAE con las cifras de aprobación de los demás estudiantes que cursaron Calculo I, y se pudo observar que las cifras son similares o superiores a favor del programa de acompañamiento. Mostrando así que el programa es un contexto apropiado para la investigación, no solo por su metodología sino también por sus resultados sobresalientes.

Fase 2. Diseño de instrumentos para la identificación inicial de habilidades del pensamiento variacional. Teniendo en cuenta el desarrollo del programa de tutorías, se reestructuraron los formatos empleados por los tutores para llevar el seguimiento de sus alumnos beneficiarios, y así para el primer semestre de 2015 se diseñó un formato de seguimiento individual por sesión que cuenta con una parte cualitativa donde los tutores describen la actividad realizada, las fortalezas y debilidades, y el trabajo complementario, seguido de una parte cuantitativa donde el tutor valora los descriptores de las habilidades asociados a los procesos matemáticos. Un segundo instrumento fue una prueba simulacro del examen 
final de cálculo I.

Fase 3. Trabajo de campo 1. Esta fase se subdivide en cuatro subfases:

i. Implementación del formato de seguimiento individual en el primer semestre de 2015 para que los tutores llevaran el seguimiento de sus estudiantes.

ii. Recolección y análisis de la información obtenida. Se identificaron los procesos más valorados por los tutores y a su vez se hicieron algunas correcciones en el instrumento, reportado en Santamaría y Parada (2015).

iii. Implementación del formato de seguimiento en el segundo semestre de 2015 e invitación a los 107 estudiantes que finalizaron el proceso tutorial para la aplicación de la prueba simulacro; 55 presentaron la prueba.

iv. Análisis de información. se tomó una muestra de estudiantes que cumpliera con dos criterios: haber presentado la prueba simulacro y contar con el formato de seguimiento individual debidamente diligenciado con al menos el 80\% de las sesiones (en total se realizan 15 sesiones). El tamaño de la muestra fue de 26 estudiantes. A continuación se muestra un resumen de resultados con respecto a las habilidades del proceso procedimental.

\section{Resultados sub-fase iv}

Se identificó de manera general, que los procedimientos de tipo numérico son los más trabajados con un promedio del 88,5\% del total de los estudiantes, mientras que los procedimientos métricos son los menos valorados con un porcentaje promedio del 40,4\%, y se corroboró porque las guías de trabajo no incluyen el uso de instrumentos específicos para medir, y no hay espacio para la exploración física, aunque algunos de los tutores usan software de geometría dinámica para simular situaciones. Los procedimientos geométricos y analíticos tuvieron un porcentaje promedio de $75 \%$ y $68,8 \%$ respectivamente.

Con respecto a su desempeño durante las sesiones, se agruparon las calificaciones en tres subgrupos (2 a 2,9; 3 a 3,9; 4 a 5 ) y más o menos la mitad de los indicadores son valorados con una calificación inferior a 3, luego suponemos que algunos de los estudiantes continúan teniendo dificultad. Por otra parte, se observó en general una valoración sobresaliente de los estudiantes ya que para el $87,5 \%$ de los indicadores el porcentaje en el subgrupo 3 a 3,9 es superior a los otros dos subgrupos.

Con respecto a la prueba simulacro, el 69,2\% de los estudiantes empleó sin dificultad procedimientos de tipo gráfico para expresar una imagen visual de los conceptos $\left(F_{5}\right)$ de función par e impar para refutar un enunciado, y el 73,1\% de los estudiantes no presentaron mayor dificultad para realizar correctamente cálculos como también para transformar expresiones algebraicas desde una forma hasta otra en el proceso de hallar el límite de una función. Sin embargo, los estudiantes tuvieron dificultades en la situación de optimización y razón de cambio, que se reflejó principalmente por la dificultad para modelar la situación de cambio a través de las funciones $\left(F_{8}\right)$ y establecer correctamente la interdependencia de las magnitudes variables $\left(F_{13}\right)$. Esto último a la luz de los porcentajes del formato de 
seguimiento no concuerda, porque ninguno de los estudiantes valorados en el indicador $F_{8}$ están en el subgrupo de calificación de 2 a 2,9, es decir, que no hay dificultad en ellos según los tutores, pero si observamos el promedio de notas para ese indicador es 3,5 luego no hay una apreciación sobresaliente en este indicador de los procedimientos analíticos.

Fase 4. Trabajo de campo 2. Después de revisar la información de cada estudiante de la muestra, se preseleccionaron tres posibles casos de estudios, que además de los dos instrumentos mencionados en la fase anterior, tienen en el sistema un reporte de la prueba diagnóstico inicial y participación en el curso de pre-cálculo. No obstante, en este documento reportaremos sobre un solo sujeto de estudio llamado Camilo, quien antes de las tutorías del semestre 2015-II presentó la prueba diagnóstico inicial, participó en el curso de pre-cálculo y presentó la prueba diagnóstico final; y después de la tutorías presentó el parcial final de la Escuela de Matemáticas, como se puede observar en la línea del tiempo de la Figura 2.

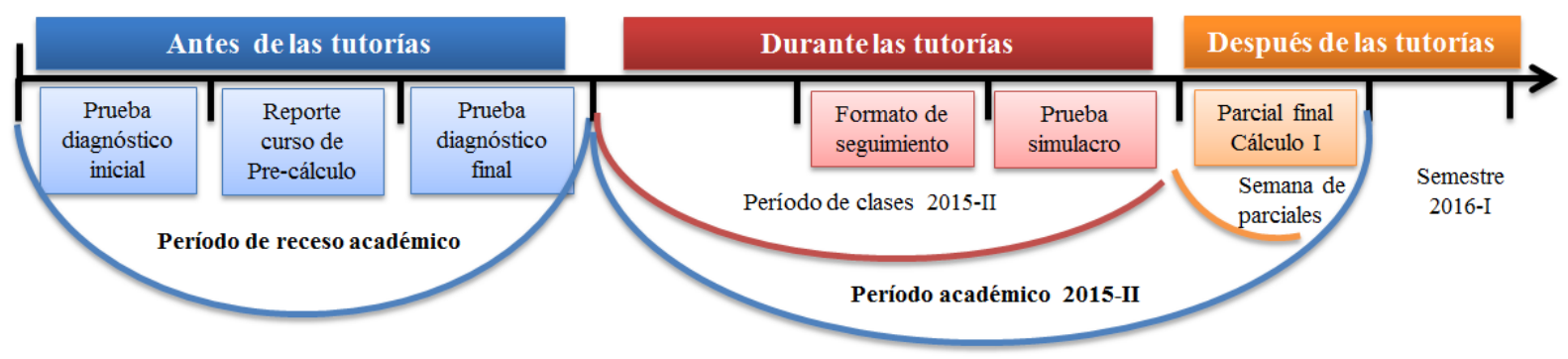

Figura 1: Línea del tiempo: caso de estudio Camilo

Resaltamos que Camilo es un estudiante egresado de una institución educativa oficial que ingresó a la UIS a la carrera de ingeniería mecánica. Dentro del programa de tutorías tuvo una tutora auxiliar; esta modalidad de tutor significa que la tutora aprobó el curso de didáctica del Cálculo y ha sido tutor auxiliar mínimo una vez. Por último, el estudiante aprobó el curso de Cálculo I en su primer semestre con una nota de 4,0 y actualmente se encuentra matriculado en el curso de Cálculo II.

Fase 5. Análisis de datos. Se analizarán los datos recolectados desde la interpretación de la tutoría como una ZDP y sus elementos, para identificar las habilidades desarrolladas por los casos de estudio desde el proceso procedimental, categorizándolos en antes, durante y después del proceso tutorial. Actualmente nos encontramos analizando los resultados de la prueba diagnóstico inicial y algunas de las sesiones de tutoría que presentamos en este documento.

Fase 6. Reporte de Resultados. Se espera al final del proceso dar respuesta a la pregunta de investigación y así contribuir al desarrollo teórico de la Educación Matemática, en particular de la línea de investigación en Didáctica del cálculo y además contribuir con el fortalecimiento de la estructura curricular del programa de tutorías, es decir, hacer una caracterización más detallada de los alcances del programa en relación al Cálculo Diferencial. 


\section{Procedimientos en la resolución de problemas en Cálculo Diferencial}

Los instrumentos del orden cronológico mostrados en la Figura 2 serán usados para presentar el análisis de los procedimientos de Camilo. Es decir, que las pruebas diagnóstico y el reporte del curso de pre-cálculo serán considerados en el apartado antes del proceso tutorial; mientras que el formato de seguimiento por sesión y la prueba simulacro serán presentados en el apartado el proceso tutorial, y en el apartado después del proceso tutorial consideraremos el parcial final de Cálculo I. En cada una de la subsecciones presentaremos los hallazgos más relevantes y algunas conclusiones basadas en la taxonomía de los procedimientos ([3]) y la ZDP de Vygotsky.

\subsection{Antes del proceso tutorial}

La prueba basada en las nociones del Cálculo Diferencial tiene, actualmente, 11 puntos que se contestan en la plataforma de la página de la Universidad y una situación que desarrolla en una hoja de procesos.

Dado que Camilo participó en el curso de Pre-cálculo tuvo que presentar la prueba diagnóstico en dos ocasiones, antes de iniciar el curso y después de él. La calificación promedio en la primera ocasión fue 3.0, en donde obtuvo la calificación máxima en 6 de los 13 puntos, y de acuerdo al sistema de calificación el estudiante quedó en nivel "medio". En esa ocasión la hoja de procesos tenía dos situaciones por eso en total se valoraron 13 puntos.

En el problema 4, Camilo obtuvo la máxima calificación y de acuerdo a [3] esta situación sobre la empresa láctea requiere de procedimientos analíticos ya que debe identificar y determinar las expresiones algebraicas que modelan el desagüe de los tanques A y B en función del tiempo a partir de la gráfica de las funciones y la tabla de valores; entonces la respuesta del estudiante muestra que quizás tiene algunos dominios relacionadas con los procedimientos analíticos, como $F_{6}$ y $F_{13}$ (Tabla 1 ). Sin embargo, la autora resalta en sus conclusiones que lo estudiantes de nuevo ingreso a la universidad tienen dificultad para "Simbolizar una relación funcional apoyándose en el análisis de los datos del problema", por lo tanto tenemos la hipótesis de que el estudiante es una excepción a la conclusión propuesta por la autora, que reforzamos con el resultado de la prueba diagnóstico final donde obtuvo la misma calificación; además su puntuación promedio fue de 3,8 clasificándose en el nivel "medio alto".

En la situación 1 de la hoja de procedimientos, en donde se debe calcular la distancia total que recorre una pelota lanzada hacia arriba antes de quedar en reposo, el estudiante no obtuvo la calificación máxima. En general, él realizó procedimientos de tipo aritmético y analítico: planteó la suma de los términos que modelan los rebotes de la pelota, entonces entendió el cambio que se produce en la variable altura, realizando una representación gráfica de la situación (recuadros verdes, Figura 2). También se evidencia que comprendió que la situación planteada involucra procesos infinitos, en la construcción del conjunto de alturas y su notación con puntos suspensivos (recuadro azul, Figura 2), no obstante tuvo dificultad para analizar correctamente la convergencia de la suma (recuadro rojo, Figura 2), que era 20 metros en realidad, entonces de acuerdo a la taxonomía de los procedimientos no tuvo habilidad para $F_{7}$ (Tabla 1). Y como lo explica [3]: 
Esto es consecuente de la dificultad para emplear un sistema numérico adecuado para representar el cambio y la variación del fenómeno, de manera que permita identificar una regularidad que lleve a una generalización y a la vez a analizar una tendencia en los datos. (p. 81)

Esta dificultad se evidencia también en el problema 10 sobre el viajero que debe desplazarse desde la ciudad A hasta la ciudad B, luego concluimos que el estudiante tiene dificultades con los procesos infinitos al inicio de su proceso universitario.

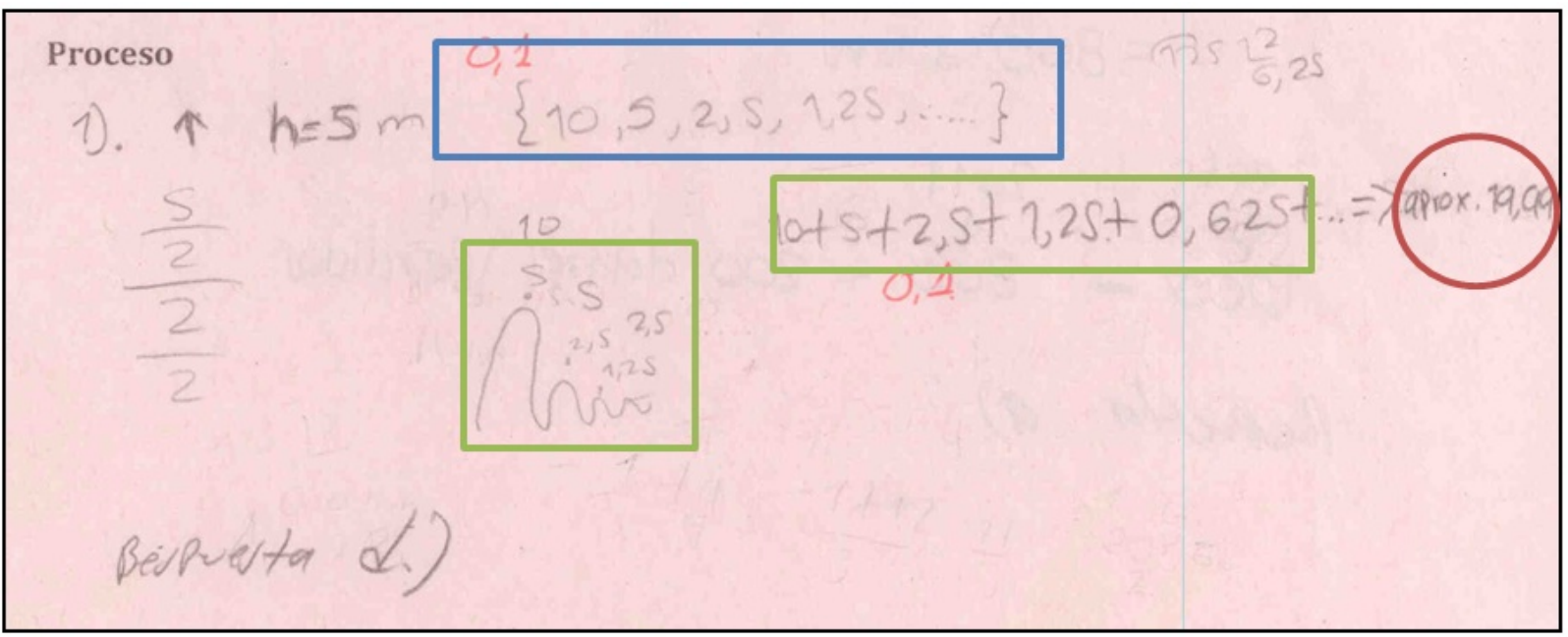

Figura 2: Procedimiento de Camilo a la situación 1, Hoja de procesos

Ahora bien para complementar el análisis inicial de Camilo y poder determinar su Zona de Desarrollo Real, miremos qué reportó la profesora de Pre-cálculo con respecto al desempeño de este estudiante:

... No presentó dificultades con las funciones y sus distintas representaciones, tampoco en la realización de procesos algebraicos, sin embargo, en la noción de límite sí hubo dificultad y como todos en el proceso de modelación. En las últimas clases mejoró su participación, además fue de los pocos que afrontó la pregunta de cómo se relacionan la variable, de una manera numérica y ayudó con eso a los demás estudiantes a buscar esas relaciones y salir de lo cualitativo. Su avance fue positivo y significativo. (Tomado del informe final del curso de pre-cálculo 2015-II)

Luego se observa para los procedimientos analíticos la habilidad en relación a las funciones y sus diferentes representaciones, no obstante representar situaciones de cambio se le dificultó a pesar de poder determinar las variables de una situación y establecer correctamente su interdependencia; además tuvo dificultades con el límite que está relacionado con los procesos infinitos, además en entrevista con él reconoció que se le dificultó representar algunas funciones como parte entera piso y modelar con ellas las situaciones que se planteaban en el curso, así como calcular e interpretar las derivadas. Por lo tanto, Camilo entró desarrollando acciones con cierta eficiencia pero no del todo dominadas. 


\subsection{El proceso tutorial}

Este apartado lo dividimos en dos partes dada la variedad de información y su orden en la línea del tiempo (Figura 1) para una mejor comunicación del proceso procedimental de Camilo en interacción con el par académico aventajado.

\subsubsection{Inicio y desarrollo del proceso tutorial}

En la primera sesión el estudiante presentó un examen diagnóstico elaborado por la tutora sobre los contenidos iniciales de pre-cálculo; de acuerdo al reporte de esa sesión se identificaron dificultades en procedimientos analíticos como traducir de una a otra de las distintas representaciones de una función, relacionar las expresiones algebraicas y gráficas empleando sus propiedades, y determinar procesos infinitos que subyacen en las notaciones decimales. Y por otro lado se resaltó el dominio en los procedimientos de tipo aritmético y en transformar expresiones algebraicas en el ejercicio de simplificar algunas expresiones. Este reporte corrobora algunas dificultades y habilidades de Camilo identificadas en la etapa anterior. Sin embargo, no se reportó habilidad para relacionar las expresiones algebraicas y gráficas empleando sus propiedades $\left(F_{6}\right)$ para cierto tipo de funciones planteadas en el examen.

Analizamos el seguimiento realizado al proceso procedimental a lo largo de tres sesiones en donde el contenido principal era límites; inicialmente la tutora manifiesta dentro del formato de seguimiento que el estudiante tuvo dificultades en la forma básica de determinar el límite de funciones, su noción no era clara, y la tutora lo atribuyó a que el estudiante "no preparó clase", y ella lo confirma con lo escrito en el trabajo complementario ya que le pide repasar los talleres trabajados y realizar nuevos ejercicios. En este punto desde la teoría de Vygotsky, la tutora está propiciando en el estudiante el proceso intrapsíquico porque debe hacer una interiorización de lo trabajado en la sesión como proceso interpsíquico, este último realizado con la tutora al depender de ella en la solución de los problemas. Recordemos que para Vygotsky la interacción social constituye el medio principal de desarrollo intelectual, y la tutoría como espacio de interacción entre pares académicos es un tipo de interacción social.

Ya en la siguiente sesión la tutora evidencia que el estudiante trabajó independientemente porque planteó dudas nuevamente sobre los límites, ratificando que aún se encuentra en una zona de desarrollo próximo con respecto a la solución de problemas sobre dicho tema, específicamente no comprendía cómo aplicar correctamente la definición formal de límite y lo pudo hacer con ayuda de la tutora, quien utilizó el software GeoGebra como herramienta de mediación para representar algunas funciones y verificar el límite en cada caso. Ya en la última sesión sobre límites, la tutora nuevamente utiliza a GeoGebra como herramienta para mirar límites de distintas funciones, creando en ese sentido un andamiaje en donde el tutor controla los elementos de la tarea y posibilita su desarrollo. Puntualmente ya no presenta dificultad en determinar los límites de las funciones una vez representados gráficamente por lo que podemos suponer que eso explica porque la tutora asignó una nota de 4,0 al indicador $F_{5}$ (Tabla 1).

Con el ejemplo anterior evidenciamos algunos de los elementos teóricos de la ZDP en el 
desarrollo de habilidades relacionadas con procedimientos analíticos. No obstante, en varias de las sesiones posteriores a éstas se identificó que la principal herramienta de mediación de la tutora, además de los ejemplos, fue el uso de Geogebra. Otro aspecto relevante fue que el espacio de la ZDP se extendió fuera de las sesiones de tutorías ya que la tutora reporta en los formatos de seguimiento que algunas de las actividades complementarias fueron socializadas por medio de redes sociales en las que el estudiante hacia llegar las inquietudes al tutor. Con respecto a las habilidades del proceso procedimental la tutora reporta en las últimas sesiones que superó varias de sus dificultades en el manejo de límites y derivadas, y que sin embargo las dificultades están en la interpretación de algunos de los enunciados, entonces a pesar de tener un avance no se identifica un domino total del indicador $F_{8}$ (Tabla 1).

\subsubsection{Finalización del proceso tutorial}

Para el semestre 2015-II la coordinación de ASAE junto con nosotras diseñamos un examen que se denominó prueba simulacro con el fin de obtener información adicional independiente de las valoraciones de los tutores, y en términos de la teoría de la ZDP identificar y describir el nuevo nivel de desarrollo alcanzado. A continuación presentamos un punto del simulacro.

En el punto 4.a. el objetivo era determinar la rapidez con que sube el nivel del agua en una determinada profundidad de un canalón con extremos verticales en forma de trapecios isósceles. Se espera que el estudiante elabore procedimientos de los cuatro tipos. Inicialmente debe representar el volumen de la figura y posterior a eso determinar las variables de la situación para modelar la expresión del volumen en función de la profundidad del agua. Esta última acción requiere de elaborar y desarrollar un procedimiento intermedio que es establecer apropiadamente la interdependencia de las magnitudes para después derivar la función volumen con respecto al tiempo y reemplazar por la información dada para finalmente calcular lo pedido.

En el procedimiento realizado por Camilo se evidencia su intento por relacionar las dos variables que son la base mayor del trapecio y la altura del agua y para ello establece una proporción de los lados correspondientes de dos triángulos semejantes, que es una sección del trapecio (recuadro verde, Figura 3). Aquí se nota que usó propiedades de figuras para determinar la interdependencia cuantitativamente.

No obstante, a la hora de modelar la función en términos de la variable altura el estudiante comete un error y no plantea correctamente el volumen de la figura 3D (recuadro rojo, Figura 3), principalmente porque hay dificultad en la interpretación y representación del área del trapecio, que a su vez, le impide denotar correctamente la base mayor en función de la altura, luego Camilo sólo consideró un triángulo mientras que la figura del trapecio está formada por dos de esos triángulos y un cuadrado de lado $1 \mathrm{~m}$. Ya en la parte final procede ordenadamente a calcular la rapidez con que sube el nivel del agua en el instante en que la profundidad es de $\frac{1}{4} m$.

En general durante el trabajo con la tutora, Camilo superó algunas dificultades y logró, por ejemplo, emplear procedimientos de tipo gráfico para expresar una imagen visual de una relación variacional que le permiten modelar situaciones de cambio. Además la mediación 


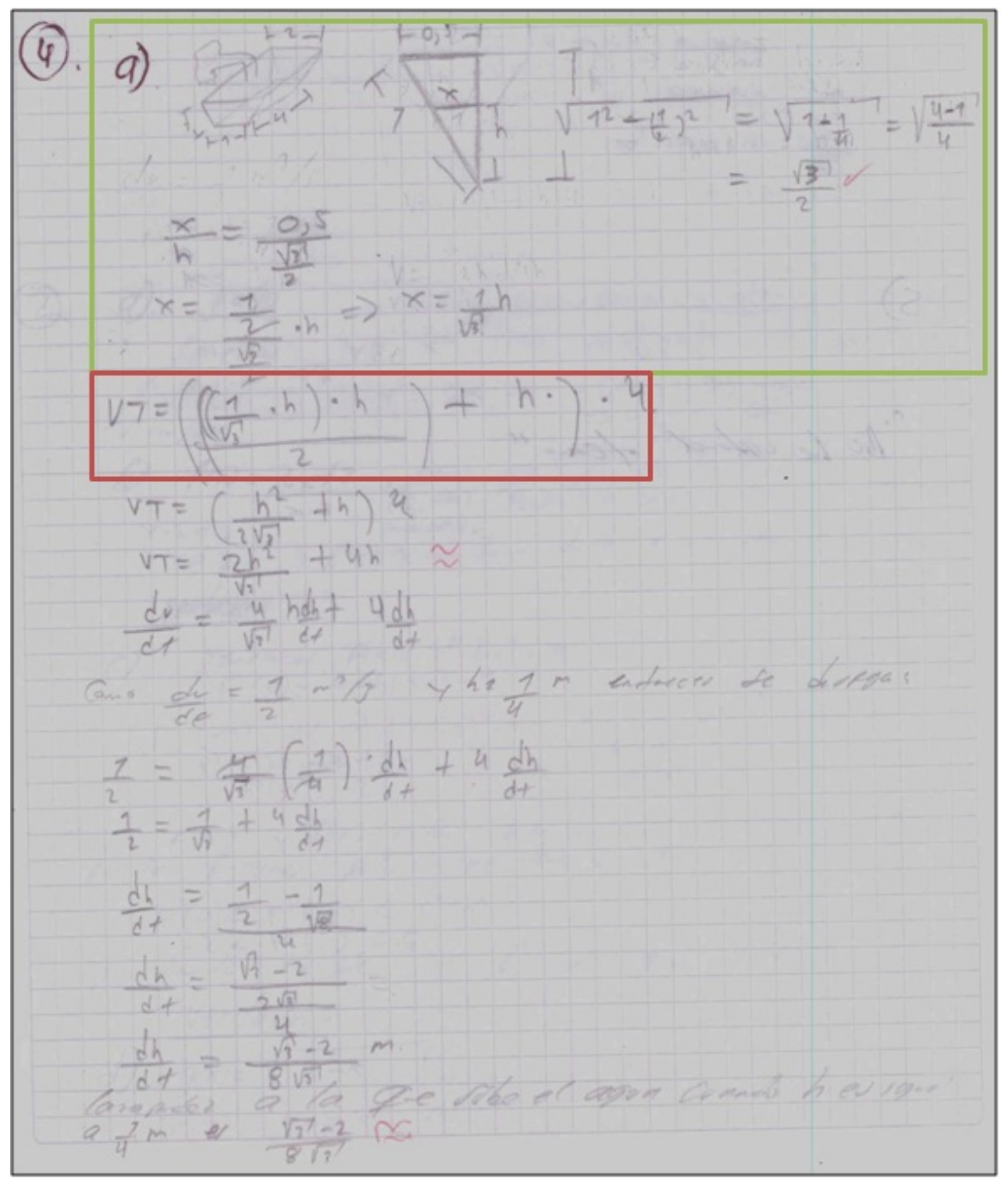

Figura 3: Procedimiento de Camilo en el punto 4.a. Prueba simulacro

hecha por la tutora con GeoGebra le permitió comprender el porqué de un límite de una función cuando el valor $\mathrm{x}$ tiende a un valor. Es de resaltar que en toda su trayectoria el estudiante no ha presentado dificultades en los procedimientos aritméticos.

\subsection{Después del proceso tutorial}

Durante las semanas de exámenes finales la Escuela de Matemáticas diseña el último parcial de Cálculo I. A continuación analizaremos los procesos expresados por Camilo en el desarrollo de este último parcial para en términos de la teoría de la ZDP dar una idea el nuevo nivel de desarrollo alcanzado.

El primer punto del examen, ver Figura 4, evalúa el cálculo y la comprensión de la derivada como razón de cambio de un fenómeno variacional. Donde el estudiante debe elaborar y realizar procedimientos de los cuatro tipos planteados en la taxonomía de [3]. 
Ahora bien en el procedimiento de Camilo a la pregunta (a), Figura 5, podemos observar que identifica y representa a cada una de las razones de cambio con la notación apropiadamente (recuadro verde, Figura 5), y a su vez determina las magnitudes variables y la constante para expresar el volumen del tanque en función de ellas (Volumen $=h \times 5$ pies $\times x$ ) entonces está usando propiedades de una figura 3D.

1. (12 $1 / 2 \mathrm{pts})$ Como se muestra en la figura, un tanque rectangular de agua de 5 pies de ancho está dividido en dos tanques por medio de una separación que se mueve en la dirección indicada a razón de $1 \mathrm{pulg} / \mathrm{min}$ cuando al tanque frontal se bombea agua a razón de $1 \mathrm{pie}^{3} / \mathrm{min}$.

(a) ¿A qué razón cambia el nivel del agua cuando el volumen de agua en el tanque frontal es de 40 pies $^{3}$ y $x=4$ pies?

(b) En ese instante, el nivel del agua ¿sube o baja?

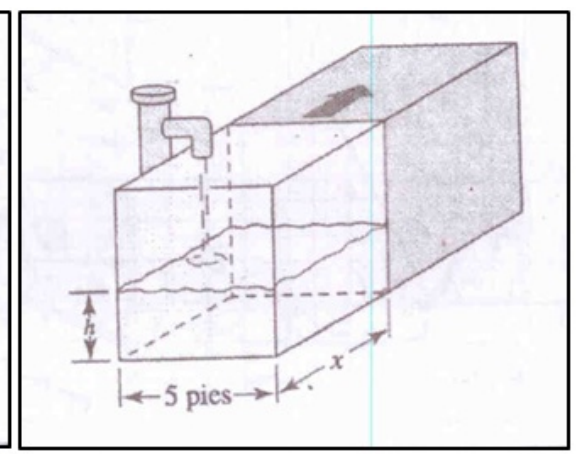

Figura 4: Enunciado problema 1, examen final

Posteriormente hace correctamente la conversión de unidades antes de continuar y determina que la variable $x$ cambia con respecto al tiempo a razón de $\frac{1}{12}$ pies $/$ min. Luego, retoma la expresión que representa el volumen del tanque y deriva con respecto al tiempo como se observa en el recuadro azul, Figura 5. Lo interesante en esta parte es que el estudiante enuncia los valores de las variables en un determinado instante y a partir de ellas deduce el valor de $h$, es decir que elabora un procedimiento para hallar el valor de una de las variables que necesita para continuar con el siguiente paso.

Una vez identificados todos los valores simplemente reemplaza y además comunica su siguiente acción y procede a solucionar la ecuación en donde la incógnita es la razón de cambio del nivel del agua, sin embargo, al sumar la unidad con la fracción (ver recuadro rojo, Figura 5) no realizó correctamente el algoritmo y por el resultado en el siguiente renglón se identifica que asignó al 6 como denominador de 1 y 5 arbitrariamente por lo cual obtuvo $\frac{4}{120}$, donde en realidad era $\frac{1}{120}$ para el valor de la razón de cambio del nivel del agua, este error cometido llama la atención porque en toda su trayectoria no había registrado dificultades para operar números fraccionarios, de hecho siempre presentó dominio en los procedimientos aritméticos, luego podemos atribuir el error a una falta de concentración en el parcial.

La mayoría de los procedimientos fueron ejecutados apropiadamente mostrando un dominio mayor en la modelación de esa situación para calcular lo solicitado, recordando que al iniciar su proceso presentaba dificultad en generar expresiones algebraicas a partir del enunciado.

Con respecto a la pregunta (b) se debe interpretar la información obtenida en el punto anterior, en donde recordemos que la razón de cambio calculada por el estudiante fue $\frac{1}{30}$ pies/min y el estudiante hace una comparación de las razones de cambio de la profundidad y el nivel del agua y argumenta que como $d x / d t$ es mayor que $d h / d t$ entonces el nivel del agua baja, lo cual es incorrecto porque independientemente de esta relación de mayor o menor lo que debe importar es que la razón de cambio del nivel de agua es positiva, luego 


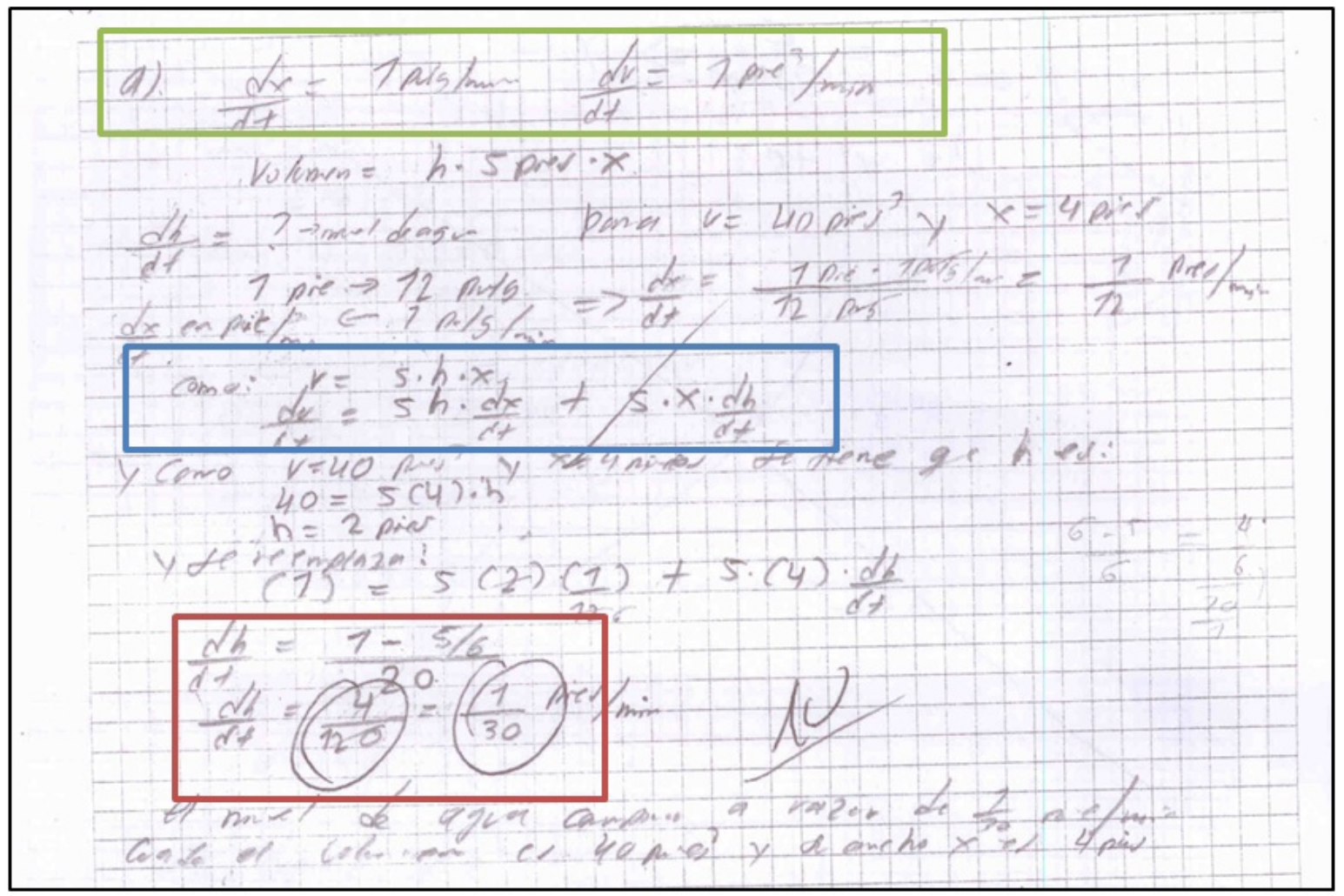

Figura 5: Respuesta a la pregunta 1.a, examen final

los valores van aumentando a una razón más pequeña que la de la otra variable pero ambas con pendiente positiva.

Entonces en este punto el estudiante tuvo dificultad para interpretar el signo de la razón de cambio y asociarlo con el valor de la pendiente de la tangente a la función ([11]). En las dificultades que tuvo notamos que los obstáculos que las provocaron son conocimiento previamente adquirido y no falta de conocimientos ([4]).

A continuación presentamos conclusiones acerca de la ZDR de Camilo vistas desde el proceso procedimental, seguido de reflexiones sobre la interacción entre pares académicos como un tipo de ZDP.

\section{Conclusiones}

En general, Camilo comenzó con dificultades para hacer aproximaciones en procesos infinitos que, más tarde, se reflejó en la determinación de límites para algunas funciones, pero la mediación de la tutora con ayuda de GeoGebra permitió que superara estas dificultades y a su vez la representación gráfica favoreció estudiar nuevas funciones que él antes no sabía cómo representar. A su vez, al iniciar el proceso presentó dificultad en generar expresiones algebraicas a partir del enunciado, sin embargo, en la mayoría de los procedimientos, evidenciados tanto en el simulacro como en el parcial final, fueron ejecutados apropiadamente mostrando así un dominio mayor en la modelación de esas situaciones de cambio para al 
final calcular lo solicitado.

No obstante se identificó una dificultad para interpretar algunos de los enunciados; esta dificultad se relaciona más con el proceso de comunicación pero afecta directamente en la elaboración y ejecución de los procedimientos. Por otro lado, siempre se evidenció un dominio sobre las operaciones con números reales a pesar de haber cometido un error en el examen final, que pudo ser por falta de atención.

Analizando el trabajo entre pares académicos que aquí se presentó, teniendo en cuenta que uno es más aventajado que el otro, y desde la teoría de Vygotsky que plantea que la interacción social constituye el medio principal de desarrollo intelectual, se puede decir que el programa de acompañamiento es un espacio propicio para el desarrollo en matemáticas de aquellos estudiantes que presentan dificultades particularmente en cálculo diferencial. Además se evidenció que el trabajo entre pares académicos como una forma de ZDP se puede extender fuera de las sesiones de tutorías a medios virtuales como lo son las redes sociales.

\section{Referencias}

[1] Artigue, M., Enseñanza y aprendizaje del análisis elemental: Qué se puede aprender de las investigaciones didácticas y los cambios curriculares. Revista Latinoamericana en Matemáticas Educativa. 1 (1), 40-55 (1998).

[2] Baquero, R., La Zona de Dearrollo Proximo y el analisis de las prácticas educativas, en Vigotsky y el aprendizaje escolar, Buenos Aires: Aique, segunda edición, 137-159.(1997).

[3] Barajas, C., Elaboración, Comparación y Ejercitación de Procedimientos: Una mirada desde la Resolución de Problemas que implican Fenómenos de Variación, Tesis de maestría, Instituto Politécnico Nacional, (2015).

[4] Brousseau, G., Les obstacles épistémologiques et la didactique des mathématiques, Construction des savoirs, 41-63, Ottawa: CIRADE (1989).

[5] Davis, R. B., The Interplay of Algebra, Geometry, and Logic, Journal of Mathematical Behavior, 7, 9-28 (1988).

[6] Dreyfus, T., Advanced mathematical thinking. En Nesher, P. y Kilpatrick, J. (Eds), Mathematics and cognition. Cambridge: Cambridge University Press, 113-133 (1990).

[7] Fiallo, J. \& Parada, S., Curso de pre-cálculo apoyado en el uso de geogebra para el desarrollo del pensamiento variacional. Revista Científica, Bogotá, Colombia, 20, 56-71. ISSN 0124-2253 (2014).

[8] Guitert, M. y Giménez, F., El trabajo cooperativo en entornos virtuales de aprendizaje, en Duart, J.M. y Sangrà, A. (Eds.), Aprender en la virtualidad. 113-134, Gedisa, Barcelona (2000). 
[9] Hitt, F., Dificultades en el aprendizaje del cálculo. En J. C. Cortés y F. Hitt (Eds), Reflexiones sobre el aprendizaje del cálculo y su enseñanza. México (2005).

[10] Ministerio de Educación Nacional, (MEN), Lineamientos curriculares en matemáticas. Bogotá: Magisterio (1998).

[11] MEN, Estándares básicos de competencias en Matemáticas. (2006). Recuperado de http://www . eduteka.org/pdfdir/MENEstandaresMatematicas2003.pdf

[12] Miranda, A., Peer tutoring: aprendiendo entre estudiantes. Médica UIS, 23, 7-8 (2010).Recuperado de http://revistas.uis.edu.co/index.php/ revistamedicasuis/article/view/979/1341

[13] NCTM, Principios y Estándares para la Educación Matemática. Traducción de M. Fernández (Traducción de la versión del 2000 del NCTM), SAEM Thales, Sevilla (2003).

[14] Rodríguez González, D., El diagnóstico de la amplitud de la zona de desarrollo próximo: Una experiencia cubana, Revista Psicoespacios, 7 (11), 307-347 (2013). Recuperado de http://revistas.iue.edu.co/index.php/Psicoespacios

[15] Rueda, N., Parada, S. \& Fiallo, J., Habilidades inherentes al pensamiento variacional de estudiantes de nuevo ingreso a la Universidad. Reporte de investigación presentado en la XIV Conferencia Interamericana de Educación Matemática, Mayo 3 al 4 en Tuxtla Gutiérrez, Chiapas, México. (2015). Recuperado de http://xiv.ciaem-iacme.org/ index.php/xiv_ciaem/xiv_ciaem/paper/viewFile/900/375

[16] Tall, D. (Ed), Advanced Mathematical Thinking, Dordrecht: Kluwer (1991). 